\title{
La frecuencia de corpus como criterio para nivelar colocaciones léxicas
}

\section{Corpus Frequency as a Criterion for Grading Lexical Collocations}

Marcos García SAlido [marcos.garcias@udc.es]

Universidade da Coruña, España

\begin{abstract}
RESUMEN
Este trabajo se propone examinar la validez de la frecuencia como criterio para ordenar las colocaciones que se deben tratar en los sucesivos niveles de aprendizaje del español. Para ello se han estudiado los siguientes aspectos: (i) la opinión de profesionales de la enseñanza de español como lengua extranjera/segunda con respecto a los criterios que ellos emplearían para nivelar vocabulario; (ii) las propuestas de dichos profesionales al respecto; (iii) su intuición sobre frecuencia colocacional; y (iv) el distinto grado de dificultad que presentan a los aprendices colocaciones clasificadas en distintos niveles según su frecuencia. Los datos para valorar estas cuatro dimensiones se han obtenido mediante cuestionarios presentados a profesores y aprendices de español.Los resultados sugieren que los profesionales de la enseñanza consideran la frecuencia un criterio importante para secuenciar el vocabulario y sus propuestas coinciden de forma notable con una nivelación basada en la frecuencia. Los resultados con respecto a la intuición de los profesores sobre frecuencia colocacional y a la correlación entre el nivel asignado a las colocaciones y la dificultad que presentan a los aprendices son menos concluyentes.
\end{abstract}

\section{Palabras Clave}

colocaciones; enseñanza de español como lengua segunda/extranjera; nivelación; frecuencia

\begin{abstract}
This article aims at examining the validity of using frequency as a criterion for assigning Spanish collocations to successive levels of proficiency. To that end, the following aspects have been studied: (i) the opinion of teachers of Spanish as a foreign/second language as to the criteria that should be used to grade vocabulary; (ii) the proposals of such professionals in that regard; (iii) their intuition on collocational frequency; and (iv) the degree of difficulty that collocations graded at different levels pose to learners. The data needed in order to explore these four dimensions have been collected by means of questionnaires administered both to teachers and learners of Spanish. The results suggest that teaching professionals regard frequency as an important criterion for grading vocabulary and their proposals coincide to a considerable extent with frequency-based grading. The findings regarding teachers' intuition on frequency as well as the correlation between frequency-based grading of collocations and collocation difficulty are less conclusive.
\end{abstract}




\section{KEYWORDS}

collocations; teaching of Spanish as a foreign/second language; grading; frequency

RECIBIDO 2016-09-30; ACEPTADO 2016-12-30

Este trabajo ha sido posible una ayuda a la investigación posdoctoral (POS-A/2013/191) concedida por la Xunta de Galicia. El autor desea expresar su agradecimiento a los centros donde se le permitió pasar los instrumentos de recogida de datos (Centro de Lenguas de la Universidade da Coruña, Cursos Internacionales de la Universidade de Santiago de Compostela y Universidad de La Rioja), así como a Margarita Alonso Ramos y a los evaluadores de Études romanes de Brno por su atenta revisión.

\section{Introducción}

Al tratar el papel del léxico en la enseñanza de una lengua segunda o una lengua extranjera (en adelante L2/LE) surge periódicamente el problema de cuál trabajar de forma explícita en cursos, textos, programaciones, etc. y de en qué fase del aprendizaje incluir un determinado repertorio de vocabulario. Una solución que aparece también de manera recurrente consiste en apelar a la frecuencia léxica. Por lo que se refiere al español, hace casi un siglo aparecía el primer listado de vocabulario frecuente (Keniston 1920) con la finalidad clara de ser aplicado a la enseñanza de la lengua. Seguirían otras obras de características similares (para una revisión mucho más detallada, vid. Izquierdo 2004: 321-385), hasta llegar al más reciente diccionario de frecuencias de Davies (2006), basado en un extenso corpus en formato electrónico y que se presenta como una propuesta de vocabulario "nuclear" (core vocabulary) para aprendices de español. También como un listado de vocabulario, ya no organizado por frecuencia, sino directamente nivelado de acuerdo con el sistema del Marco Común Europeo de Referencia para las lenguas (Consejo de Europa 2001; en adelante MCER) pueden verse ciertas secciones del Plan curricular del Instituto Cervantes (1997-2016; en adelante PCIC), en concreto las tituladas Nociones generales y Nociones específicas. La frecuencia léxica vuelve a desempeñar aquí un papel clave en la selección, tal como se indica en la obra citada: "Como criterios de selección, siempre partiendo de la apreciación intuitiva basada en la experiencia docente, han primado la frecuencia y la rentabilidad comunicativa"1.

Los mencionados listados del PCIC no incluyen solo vocabulario univerbal. De hecho, se presentan no como listados de voces, sino como listados de exponentes de nociones, esto es, de las codificaciones lingüísticas que recibe un determinado sentido o combinaciones de sentidos ${ }^{2}$.Así, nos encontramos desde unidades léxicas (ombligo, en el nivel C1) a locuciones (irse a pique [una empresa], en C2), pasando por combinaciones (dar un paseo, B1) que encajan con la definición de colocación que se adopta aquí. Precisamente, las colocaciones serán el objeto de estudio del presente trabajo.

1 http://cvc.cervantes.es/ensenanza/biblioteca_ele/plan_curricular/niveles/09_nociones_especificas_introduccion.htm

2 En el PCIC el componente nocional se define como un conjunto de "contenidos que tienen que ver, en sentido amplio, con el significado”. (http://cvc.cervantes.es/ensenanza/biblioteca_ele/plan_curricular/niveles/08_nociones_generales_introduccion.htm) 
Partiendo de una perspectiva fraseológica, una colocación es una combinación binaria de unidades léxicas tal que el hablante escoge una de ellas (la base) teniendo en cuenta exclusivamente el significado que quiere expresar, mientras que la elección de la otra (el colocativo), además de por el significado que se pretende transmitir, viene determinada por la identidad de la primera (Mel'čuk 2012: 38, 39). Volviendo al ejemplo citado arriba, dar un paseo, se hace evidente que la elección del lexema dar está condicionada por la identidad del nombre paseo cuando comparamos esta combinación con las posibilidades que se presentan para el nombre excursión. Ambos nombres transmiten un sentido similar de 'desplazamiento con fines recreativos' y, sin embargo, el lexema excursión parece rechazar la combinación con dar, prefiriendo hacer para expresar el sentido de 'realizar, llevar a cabo' (cf. ??dar una excursión y hacer una excursión). El empleo del lexema dar con el significado de 'realizar, llevar a cabo' es, pues, posible solo en ciertos contextos, dependiendo del nombre que funcione como su objeto. Si dicho fenómeno existe en los términos que se ha descrito, es razonable pensar que suponga una dificultad adicional para el aprendizaje del vocabulario de una L2/LE, toda vez que dicho aprendizaje no se limita a la adquisición de un catálogo de formas que mantienen una relación multívoca con un conjunto de significados (polisemia, sinonimia...), sino que además el aprendiz ha de acabar sabiendo qué contextos permiten la codificación de un determinado significado por medio de un cierto significante. De hecho, una serie de estudios más o menos recientes ha constatado los problemas que plantea, incluso a aprendices avanzados, la producción de colocaciones en diversos idiomas (Gilquin 2007; Nesselhauf 2004; Vincze et al. 2016, etc.).

Si la propia existencia de unidades fraseológicas (incluidas las colocaciones), así como la dificultad que comporta su dominio aconsejan que la enseñanza del léxico tenga en cuenta repertorios de vocabulario que no se limiten a listados de palabras aisladas, los criterios manejados para la inclusión y secuenciación de dichas unidades no tienen por qué ser distintos en un uno y otro caso. Así, la frecuencia de uso vuelve a aparecer una y otra vez como un criterio que ha de considerarse tanto para la elección y nivelación de colocaciones (Ferrando Aramo 2012:360-361), como en el ámbito de la fraseología en sentido más amplio (Nation 2001: 329; Martinez 2013).

El razonamiento que lleva a escoger la frecuencia como un criterio fundamental para elegir el vocabulario que se trabaja explícitamente en la instrucción de los aprendices, así como para determinar a cuál se ha de dar prioridad, descansa sobre la idea de que el léxico frecuente, por el hecho de serlo, resulta rentable (Martinez 2013: 187; Nation 2001: 13 y ss.): así, si una serie de formas se presentan en los textos de manera muy recurrente, conocerlas ayuda en gran medida tanto a descifrar dichos textos como a producir otros nuevos. Ahora bien, esta correspondencia no siempre se da y se han aducido casos relevantes donde no se produce (cf., por ejemplo, Alonso Ramos 2012; Izquierdo 2004). Se ha señalado, por ejemplo, que las palabras gramaticales, que en general presentan una frecuencia elevada, contribuyen menos que las léxicas a dotar al mensaje de contenido. También se ha apuntado que expresiones no especialmente frecuentes en una mayoría de textos pueden resultar muy útiles para un aprendiz en un contexto de uso real de la lengua meta (p. ej., tarjeta sanitaria en una estancia para aprender el idioma). Quizá estas faltas ocasionales de correspondencia entre frecuencia y rentabilidad sean el motivo por el cual en ciertas propuestas de nivelación o de selección de vocabulario se incluyen criterios adicionales, además de la frecuencia de corpus. Así, por ejemplo, el English Vocabulary Profile (Capel 2010; 2012), quizá el proyecto más ambicioso 
en este sentido, se apoya, además de en diversos listados de frecuencia, en las producciones de los propios aprendices para determinar cuál es el vocabulario (incluyendo expresiones poliléxicas) propio de los diferentes niveles distinguidos por el MCER. El diccionario de colocaciones para aprendices de italiano, DICI-A (Spina 2016), combina la frecuencia con una medida de dispersión y con la pertenencia del vocabulario en cuestión a determinados temas para asignar manualmente nivel a las colocaciones que contiene. Como último ejemplo en esta línea de investigación, Benigno et al. (2015) proponen un sistema para elaborar una lista de colocaciones fundamentales combinando diversas medidas de asociación y dispersión (la frecuencia entre ellas, que parece ser la que ofrece mejores resultados para el propósito de los autores) con la intuición de hablantes nativos sobre el "carácter fundamental" de una determinada combinación léxica.

Habida cuenta de la importancia de la frecuencia como criterio determinante en la selección y nivelación del vocabulario en general y de las colocaciones léxicas en particular, el presente trabajo se propone investigar hasta qué punto un sistema de nivelación basado en este criterio (dejando de lado otros posibles) se corresponde, por una parte, con la práctica de los docentes de ELE/L2 y, por otra, con los conocimientos de los aprendices. En concreto, se intentará responder a las siguientes preguntas:

i) ¿Qué criterios consideran los profesionales de ELE/L2 que se deben emplear para nivelar el léxico?

ii) Si los profesionales creen que la frecuencia de uso es un criterio válido, ¿podrían nivelar una lista de colocaciones del español basándose en su intuición? O formulado de otra manera: ¿qué grado de intuición tienen los profesores de ELE/L2 con respecto la frecuencia de las colocaciones del español?

iii) ¿Qué grado de correspondencia habría entre un sistema que ordene las colocaciones del español en niveles basándose en la frecuencia que estas presentan en un corpus y la nivelación propuesta por profesores de ELE/L2?

Y en lo que concierne a los aprendices de español:

iv) Partiendo de una nivelación basada en frecuencia de uso, ¿ ¿resultan las colocaciones de niveles más bajos más fáciles que las de niveles más altos? Es decir, ¿las colocaciones de niveles más bajos son conocidas por un número mayor de aprendices?

Para responder a estas preguntas en las páginas siguientes se dará cuenta de dos estudios, uno centrado en profesores de ELE/2 y otro en aprendices. Previamente se presentarán los instrumentos utilizados en cada caso y los participantes de ambos estudios (apartado 2). Tras exponer los resultados obtenidos (apartado 3) y discutir su interpretación (apartado 4), el trabajo se cierra apuntando una serie de conclusiones. 


\section{Recogida de datos y participantes en el estudio}

Para el presente estudio se ha acudido a dos tipos de datos fundamentales: los obtenidos a partir de un cuestionario a profesores de ELE, por un lado, y, por otro, los recabados de aprendices de esta lengua. En este apartado se describen los instrumentos de recolección de datos utilizados con cada uno de estos dos grupos.

Las colocaciones con sus respectivos niveles presentadas tanto a los profesores como a los aprendices se extrajeron del Diccionario de Colocaciones del español (Alonso Ramos 2004; en adelante $\mathrm{DiCE}$ ). A las colocaciones de este diccionario se les han adjudicado los niveles establecidos por el MCER (fundiendo los dos inferiores, A1 y A2, en uno único). El criterio utilizado para ello fue exclusivamente la frecuencia de uso (para más detalles sobre el procedimiento seguido, vid. García Salido y Alonso Ramos, en prensa). La información relativa a la frecuencia de las colocaciones contenidas en el DiCE fue obtenida a partir de la sección de español europeo del corpus esTenTen (Kilgarriff y Renau 2013; para más detalles sobre la asignación de frecuencia a las colocaciones del DiCE, vid. Vincze y Alonso Ramos 2013).

\subsection{Cuestionario a profesores}

El cuestionario a profesores constaba de tres partes fundamentales. La primera tenía como objetivo medir hasta qué punto el sistema de nivelación aplicado en el DiCE coincide con la que propondría un profesional de la enseñanza de ELE. En ella se pedía a los participantes que nivelasen un conjunto de 10 colocaciones de las que figuran en el diccionario de acuerdo con los niveles del MCER tal como se han asignado en el DiCE — esto es, en cinco categorías - (vid. tabla 1). Las colocaciones se presentaban en orden aleatorio.

\begin{tabular}{lc}
\hline COLOCACIÓN & NIVEL EN DiCE \\
\hline dar pena & A1/A2 \\
tener (la) culpa & A1/A2 \\
mantener una amistad & $\mathrm{B} 1$ \\
perder el respeto & $\mathrm{B} 1$ \\
causar admiración & $\mathrm{B} 2$ \\
llevarse un disgusto & $\mathrm{B} 2$ \\
cultivar una afición & $\mathrm{C} 1$ \\
paliar el dolor & $\mathrm{C} 1$ \\
expresar aprecio & $\mathrm{C} 2$ \\
alentar una ilusión & $\mathrm{C} 2$ \\
\hline
\end{tabular}

Tabla 1. Colocaciones para nivelar en el cuestionario a profesores 
La segunda parte obedecía al propósito de medir la correspondencia entre la intuición de la frecuencia colocacional de los citados profesionales y la frecuencia con la que una colocación ocurre en una muestra representativa del español. El método empleado es similar al usado en las investigaciones de Arnaud (1990), Frey (1981) y Ringeling (1984) para palabras aisladas y McGee (2006: 133 y ss.), que lo aplica a colocaciones del inglés: consiste en presentar al sujeto una lista de expresiones en orden aleatorio y pedirle que las ordene de más a menos frecuente. De manera similar a McGee se ha procurado que los ítems estuvieran separados por una distancia similar en cuanto a su frecuencia: en concreto, se ha procurado que cada una de las colocaciones elegidas tuviese una frecuencia de aproximadamente el doble que la inmediatamente inferior. Las colocaciones de esta tarea se presentan en la tabla 2.

\begin{tabular}{|c|c|c|}
\hline COLOCACIÓN & FREC/MILLÓN & DISTANCIA \\
\hline llamar la atención & 36,02 & 2 \\
\hline tener miedo & 15,66 & \\
\hline dar pena & 8,43 & y18 \\
\hline despertar interés & 4,73 & $\mathrm{x} 2$ \\
\hline merecer respeto & 2,39 & $\mathrm{x} 2$ \\
\hline sufrir dolor & 1,19 & $\mathrm{x} 2$ \\
\hline ponerganas & 0,59 & $\mathrm{x} 1,9$ \\
\hline tener afecto & 0,31 & $\mathrm{x} 1,9$ \\
\hline sentir envidia & 0,16 & \\
\hline
\end{tabular}

Tabla 2. Colocaciones para ordenar según su frecuencia en el cuestionario a profesores

La técnica es de las más sencillas que se mencionan en la bibliografía al respecto y esto en sí mismo puede verse como una ventaja. Un ejemplo de una técnica bastante más complicada puede encontrarse en Schmitt y Dunham (1999). Estos autores proporcionan a los sujetos una lista con una forma que sirve de punto de referencia, de modo que el resto de ítems ha de ordenarse en relación con dicha referencia, explicitando en cada caso cuánto más o menos frecuente es el ítem en cuestión por medio de múltiplos o fracciones (p. ej., si se estima que el ítem que se está ordenando es 10 veces más frecuente que el punto de referencia y este tiene el valor 1, a aquel habrá de atribuírsele un 10; si se estima que un ítem es tres veces menos frecuente que la referencia, se le asignará $1 / 3$ o 0,33 , etc.). Estas complicaciones añadidas hacen que sea difícil determinar si se está valorando exclusivamente la intuición sobre frecuencia léxica de los informantes o sus habilidades matemáticas, como apunta McGee (2006: 101). 
El número de ítems presentados a los informantes es similar al los ocho que presenta McGee (2006) - si bien, el autor administra cuatro listas distintas-. En el resto de estudios citados que aplican una técnica similar, el número de ítems oscila entre los doce de Frey (1981) y los treinta (Arnaud 1990). La longitud de la lista viene, asimismo, determinada por las posibilidades que permite el $\mathrm{DiCE}$ al respecto, así como por las limitaciones impuestas por haber fijado intervalos de frecuencia similares entre las colocaciones presentadas: el hecho de buscar una lista cuyos miembros son, por su frecuencia, submúltiplos del anterior y están además separados por un intervalo similar limita considerablemente las posibilidades dentro del repertorio de colocaciones contenidas en el citado diccionario. Además, es dudoso que los participantes pudieran ordenar de manera fiable repertorios mucho más grandes (uno de ellos se queja de lo dificultoso de la tarea y, a juzgar por los resultados expuestos más abajo, no puede asegurarse que todos hayan comprendido su objetivo).

La tercera parte del cuestionario pregunta directamente por qué criterios consideran los profesionales que se deberían emplear para nivelar el vocabulario que se presenta a los aprendices. La pregunta es abierta, de modo que los informantes pueden añadir las consideraciones que consideren oportunas, pero de partida se proporcionan las siguientes opciones:

(1) a. La frecuencia de uso

b. Los temas que se tratan en cada nivel

c. Repertorios de vocabulario y combinaciones léxicas ya ordenados según los niveles del $M C E R$, como los que aparecen en el PCIC

El cuestionario se difundió a través de la red, principalmente por medio de la lista de distribución Formespa ${ }^{3}$, pero también se hizo llegar a los profesionales de los centros que colaboraron en la aplicación del test de aprendices. La muestra se compone, por tanto, de respuestas de informantes autoseleccionados. En total se consiguieron 34 respuestas válidas.

\subsection{Cuestionario a aprendices}

Este instrumento fue concebido con la intención de determinar si existe algún tipo de correlación entre el nivel de una colocación, establecido a partir de su frecuencia, y la dificultad que esta presenta a los aprendices de español ${ }^{4}$. Se compone de un total de quince ítems: cada uno presenta una

3 http://formespa.rediris.es/

4 Aunque por su formato y por el hecho de que las respuestas se valoren con respecto a la norma nativa pueda asimilarse a un test, aquí se ha prescindido de esta denominación. Tal como apunta Dörney (2002: 6-7), la finalidad de un test es hacer inferencias sobre el grado de desarrollo de una determinada competencia, capacidad o habilidad de un individuo y, en el caso de una LE/L2, generalmente el grado de desarrollo de esa L2. En el presente estudio, sin embargo, el instrumento se administró a estudiantes ya nivelados por sus respectivos centros de estudio y su finalidad no era tampoco evaluar su competencia léxica o colocacional, sino la dificultad asociada a la frecuencia/nivel de determinadas colocaciones del español (aunque esta venga determinada por aquella). Por esta misma razón, determinadas expectativas con respecto al comportamiento de un buen test no tienen demasiado sentido aquí. Por ejemplo, uno de los factores que se relacionan con la fiabilidad y consistencia interna de un test es que todos sus ítems tengan un grado de dificultad similar (Fulcher y Davidson 2007: 106). Aquí son de esperar grados de dificultad distintos dependiendo de la frecuencia de la colocación presentada en cada ítem. 
colocación de un cierto nivel (hay tres por nivel), todas con la estructura verbo-objeto directo. Para cada ítem se ha construido un enunciado que fuerce el uso de la colocación meta. El aprendiz debe elegir entre el colocativo y tres distractores. Puesto que la tarea no se dirige aprendices de un mismo nivel, se ha controlado la frecuencia de las formas que se emplean en los enunciados, más allá de las de la propia colocación y los distractores: debían ser formas que correspondieran a los 5000 lemas más frecuentes del esTenTen de español europeo o bien cuyo lema estuviese incluido en Davies (2006). Con esto se perseguía minimizar los posibles problemas de comprensión causados por palabras ajenas a la colocación en cuestión.

Los distractores en cada ítem o bien son colocativos válidos para la base en cuestión extraídos igualmente del $\mathrm{DiCE}$, pero que dan como resultado una colocación incoherente en el contexto proporcionado, o bien verbos que no forman colocación con esa base pero tienen un sentido que sí encaja en el contexto, tal como ilustra el ejemplo siguiente:

(2) Una forma de a un amigo es hacerle un regalo.
a. tomar aprecio
c. coger aprecio
b. enseñar aprecio
d. expresar aprecio

Tomar y coger aprecio son colocaciones válidas en español que no son coherentes en el contexto indicado, mientras que enseñar aprecio no parece una combinación aceptable, a pesar de que el verbo pueda transmitir un sentido similar al de expresar en compañía del nombre en cuestión.

La tarea podría calificarse como de reconocimiento activo, según la terminología propuesta por Laufer y Goldstein (2004). Este tipo de tarea implica, por una parte, que el participante ha de reconocer un elemento determinado de la lengua meta (pero no recuperarlo de su memoria) y, por otra, que parte de un cierto significado para llegar a la forma que lo expresa (lo contrario, esto es, ir de la forma al significado, se ha calificado en ocasiones como una tarea de tipo pasivo).

La direccionalidad de la tarea (del significado a la forma) viene en cierto modo impuesta por la naturaleza de las colocaciones, tal como se conciben en el DiCE y en este estudio (vid. apartado 1): lo definitorio de este tipo de combinaciones léxicas es que, para expresar uno de los significados que transmite la combinación, el hablante no puede elegir cualquier lexema asociado a dicho significado en español, sino que ha de escoger entre un conjunto de posibilidades restringido por la base. Teniendo esto en cuenta, podría pensarse que la tarea idónea hubiera sido una completamente productiva, es decir, de recuperación activa. Sin embargo, con este tipo de tarea hubiera sido muy difícil controlar que las respuestas se correspondieran con el nivel deseado. Así, por ejemplo, en el propio DiCE vienen consignados los lexemas dar y causar, que, como colocativos de pena, expresan el significado 'causar'. Ahora bien, entre las colocaciones causar pena y dar pena existen diferencias notables en cuanto a la frecuencia de uso y, en consecuencia, tienen asignados niveles distintos (C1 y A1/A2, respectivamente). Si nuestro objetivo es verificar la dificultad de una colocación perteneciente a un determinado nivel, se antoja difícil crear un ítem que por su significado provoque el uso de una de las dos formas y excluya la otra. Imaginemos además que la forma meta del ítem en cuestión es dar pena y un aprendiz produce causar pena. Sería absurdo deducir de ello que desconoce la primera y penalizar su conocimiento de colocaciones de nivel A1/A2. 
El cuestionario se administró a un grupo de doce nativos para asegurar que cada ítem condujera a la respuesta deseada. Los distractores considerados respuestas válidas por parte de algún hablante nativo se sustituyeron.

Los participantes fueron aprendices de español de los niveles B1 a C2, si bien la muestra no está equilibrada en cuanto al nivel por la propia composición del alumnado de los centros a los que el autor ha podido tener acceso. En concreto, de un total de 54 aprendices solo hay cinco hablantes de nivel C1 o C2 (el 9\% del total). Las características de la muestra se presentan en la tabla 3:

\begin{tabular}{|c|c|c|c|c|}
\hline & & & \multicolumn{2}{|c|}{$\mathrm{n}$ participantes } \\
\hline \multirow[t]{7}{*}{ L1 } & chino & & \multicolumn{2}{|c|}{16} \\
\hline & coreano & & \multicolumn{2}{|c|}{2} \\
\hline & holandés & & \multicolumn{2}{|c|}{1} \\
\hline & inglés & & \multicolumn{2}{|c|}{30} \\
\hline & japonés & & \multicolumn{2}{|c|}{3} \\
\hline & lituano & & \multicolumn{2}{|c|}{1} \\
\hline & vietnamita & & \multicolumn{2}{|c|}{1} \\
\hline \multirow[t]{2}{*}{ Sexo } & mujeres & & \multicolumn{2}{|c|}{40} \\
\hline & hombres & & \multicolumn{2}{|c|}{14} \\
\hline \multirow[t]{3}{*}{ Nivel MCER } & $\mathrm{B} 1$ & & \multicolumn{2}{|c|}{31} \\
\hline & B2 & & \multicolumn{2}{|c|}{18} \\
\hline & $\mathrm{C} 1 / \mathrm{C} 2$ & & \multicolumn{2}{|c|}{5} \\
\hline \multirow[t]{2}{*}{ Edad } & media & desv. est. & mín. & máx. \\
\hline & 23,35 & 8,87 & 17 & 62 \\
\hline
\end{tabular}

Tabla 3. Datos de los participantes

Como en el caso anterior, la participación en el cuestionario fue voluntaria. El instrumento se administró en períodos de tiempo libre entre clases, en formato papel, y su realización nunca excedió los 15 minutos.

\section{Resultados}

Expongo a continuación los datos obtenidos mediante los instrumentos que se detallan en el apartado anterior, comenzando por los correspondientes a profesores de ELE para, a continuación, presentar los relativos a los aprendices. 


\subsection{Cuestionario a profesores}

Con el fin de medir la coincidencia entre el sistema de nivelación aplicado al DiCE y el criterio de los docentes de ELE, se ha hallado la correlación entre los niveles que se les asigna en el diccionario a las 10 colocaciones presentadas en el cuestionario a profesores y los propuestos por este colectivo. Para ello se han transformado los cinco niveles distinguidos en rangos (A1/A2 es el rango 1, B1 el rango 2, etc.) y se ha hallado el coeficiente de correlación $\tau_{\mathrm{b}}$ de Kendall para cada informante. Un coeficiente de 1 significaría una coincidencia absoluta entre el $D i C E$ y la propuesta del profesor, mientras que un coeficiente de - 1 indicaría que el profesor adjudica una nivelación exactamente inversa a la del diccionario (esto es, donde el $\mathrm{DiCE}$ da C2, el profesor propondría A1/ A2; para un $\mathrm{C} 1$ del diccionario, el profesor defendería un B1, etc.). La media de las correlaciones obtenidas para los profesores que participaron en el estudio es de 0,62 (desv. est. $=0,21$ ), pero la variabilidad entre unos y otros es notable. Así, algunos de los participantes en el estudio muestran niveles muy altos de coincidencia con la nivelación aplicada en el DiCE (el coeficiente más alto es de 0,88 ), mientras que otros proponen un patrón sin una correlación obvia (el coeficiente mínimo es de $-0,08$, si bien, valores tan bajos son excepcionales). La mayoría de participantes (en concreto, el 65\%) presentan coeficientes iguales o mayores a 0,60.

La segunda pregunta del cuestionario estaba dirigida específicamente a comprobar la intuición que de la frecuencia colocacional tenían los participantes: se les pedía que clasificaran una lista de nueve colocaciones de la más a la menos frecuente. Para hallar la correlación entre la ordenación de los participantes y la resultante de la frecuencia de las colocaciones se usó el coeficiente de Spearman. La interpretación del coeficiente es la misma que en el caso anterior. En este caso la media de los coeficientes es positiva, pero muy baja $(0,29)$ y la variación entre participantes mayor que en la tarea anterior (desv. est. $=0,41$ ). Como en la prueba previa, ciertos participantes tienen una intuición muy fiable acerca de la frecuencia colocacional (el coeficiente más alto es de 0,90 ), mientras que otros parecen tener intuiciones que van en la dirección opuesta a lo que se registra en el corpus utilizado (el coeficiente más bajo es de -0,78). Habida cuenta de que en esta prueba se registran casos recurrentes de correlaciones negativas con valores considerablemente bajos (hay cinco casos de coeficientes negativos, tres de ellos por debajo de -0,5), podría pensarse que alguno de los participantes interpretó mal las instrucciones y ordenó la lista en orden creciente de frecuencia (de hecho, uno se quejó de la complejidad de la tarea). Si este es el caso, la media de los valores absolutos de los coeficientes obtenidos sería más informativa: resulta así un valor ligeramente más alto $(0,45$, desv. est. $=0,21)$, pero que tampoco revela que la intuición media de los participantes sobre frecuencia colocacional sea especialmente buena.

Al contrario que en la tarea de nivelación, en esta, que mide directamente la intuición sobre frecuencia, los participantes con coeficientes por encima del 0,6 son minoría (cuatro, el 12\%) y los que tienen intuiciones realmente buenas - por encima del 0,7- constituyen un grupo muy reducido (únicamente dos participantes, el 6\%). Si tomamos como referencia los valores absolutos, asumiendo que los participantes que obtuvieron valores negativos ordenaron las colocaciones a la inversa de como se les pedía, solo seis participantes (18\%) presentan correlaciones por encima de $0,6 \mathrm{y}$, de estos, cuatro (12\%) por encima del 0,7 .

Por último, en cuanto a los criterios que los profesores participantes en el estudio creen que deben emplearse en la nivelación del vocabulario, es la frecuencia de uso la respuesta que más se 
repite, como se aprecia en la siguiente tabla (téngase en cuenta que las respuestas no se excluyen mutuamente, por lo que los diferentes porcentajes no tienen por qué sumar 100):

\begin{tabular}{lc}
\hline CRITERIO & $\mathrm{N}(\%)$ \\
\hline Frecuencia de uso & $25(73.5 \%)$ \\
Repertorios léxicos & $16(47.1 \%)$ \\
Temas de cada nivel & $14(41.2 \%)$ \\
Otros & $3(8.8 \%)$ \\
\hline
\end{tabular}

Tabla 4. Respuestas al criterio de nivelación preferido

Los tres participantes que proporcionaron respuestas distintas a las facilitadas proponían atender a combinaciones difíciles tanto desde el punto de vista léxico como desde el punto de vista gramatical, a combinaciones léxicas de uso muy reducido, tener en cuenta la influencia de la lengua materna de los aprendices y basarse en las jerarquizaciones que ofrecen manuales dirigidos específicamente a aprendices de ELE.

\subsection{Cuestionario a aprendices}

En cuanto a los datos obtenidos a partir del instrumento dirigido a aprendices, los resultados son los que se expondrán a continuación. Recordemos que la finalidad aquí era determinar si existía alguna relación entre el nivel asignado a una determinada colocación y la dificultad que presentaba para un aprendiz. A tal efecto se han calculado índices de dificultad para cada una de las colocaciones presentadas a los participantes, que no son sino la proporción de aciertos que ha tenido cada una. Estos datos se presentan en la tabla 5.

\begin{tabular}{cccc}
\hline ITEM & NIVEL & DIFICULTAD & DIF. MEDIA \\
\hline 1 & & 0,61 & 0,65 \\
8 & & 0,57 & \\
15 & A1/A2 & 0,78 & 0,71 \\
\hline 2 & & 0,65 & \\
6 & & 0,67 & 0,44 \\
13 & B1 & 0,81 & \\
\hline 4 & & 0,48 & \\
\hline
\end{tabular}




\begin{tabular}{cccc}
\hline ITEM & NIVEL & DIFICULTAD & DIF. MEDIA \\
\hline 5 & & 0,44 & 0,50 \\
9 & & 0,56 & \\
12 & $\mathrm{C} 1$ & 0,50 & 0,55 \\
\hline 3 & & 0,81 & \\
10 & & 0,37 & \\
\hline 14 & $\mathrm{C} 2$ & 0,46 & \\
\hline
\end{tabular}

Tabla 5. Índices de dificultad de los ítems del cuestionario para aprendices

Tal como se desprende de los datos, a primera vista no se aprecia una correlación obvia entre nivel y dificultad. Así, si bien uno de los ítems con una menor proporción de aciertos se corresponde con el nivel C2, tal como cabría esperar, uno con una proporción todavía menor pertenece al nivel B2; los ítems más fáciles corresponden a los niveles C2 y B1, etc. Para comprobar hasta qué punto estas dos dimensiones (nivel y dificultad) están correlacionadas se ha hallado el coeficiente de correlación Spearman entre los rangos de los distintos niveles y los derivados de las medias de los índices de dificultad correspondientes a cada nivel. De existir alguna correlación, cabría esperar que esta fuera negativa: es decir, que las colocaciones de nivel más alto obtuviesen una menor proporción de aciertos. El coeficiente obtenido es efectivamente negativo, pero no muy alto $(\rho=-0,5)$ y no significativo $(\mathrm{p}=0,45)$.

Habida cuenta de que los participantes en esta parte del estudio estaban clasificados en distintos niveles del MCER, es interesante analizar los resultados según el nivel de los aprendices en cuestión. Ahora bien, es preciso tomar ciertas precauciones a este respecto. En primer lugar, hay que tener en cuenta que el nivel de un aprendiz no se establece únicamente en función de su conocimiento del vocabulario. Es más, en el sistema de descriptores propuesto en el MCER, el vocabulario ni siquiera es una categoría que se maneje en el establecimiento de distintos niveles de dominio de una lengua, sino que parece tenerse en cuenta de forma transversal en dimensiones como las denominadas "alcance" o "corrección" (cf. MCER: 31, 32). En segundo lugar, como se apunta en el apartado 2, el conjunto de aprendices al que se ha tenido acceso en el momento de elaboración del estudio o bien realizaba cursos en instituciones diferentes, o bien había completado su instrucción formal en ELE (C1, C2). Por todo ello, se ha comprobado si había diferencias significativas entre los tres niveles de partida (B1, B2 y un único grupo C debido a la escasa participación de hablantes pertenecientes a los dos escalafones superiores) en cuanto a su actuación en el instrumento diseñado para el presente trabajo. Las medias de cada grupo (sobre una puntuación máxima de 15) se presentan en la tabla 6.

\begin{tabular}{ccc}
\hline GRUPO & MEDIA & DESV. EST. \\
\hline B1 & 8,55 & 2,62 \\
B2 & 7,39 & 4,09 \\
C & 12,8 & 1,64 \\
\hline
\end{tabular}

Tabla 6. Medias de los participantes por nivel 
Un test ANOVA determinó la existencia de diferencias significativas entre los grupos $(\mathrm{F}(2,51)=5,835, \mathrm{p}=0,00523)$ y para determinar a cuáles de ellos se debían se utilizó el post-test de Tukey, con las modificaciones propuestas por Herberich et al. (2010), puesto que no se cumplía el supuesto de homogeneidad de varianzas ( $\mathrm{p}=0,02$ en un test de Levene). Estas comparaciones revelaron el grupo $C$ difiere significativamente de los otros dos $(C-B 1 t=4,47, p<0,0001 ; C-B 2 t=4,20$, $\mathrm{p}=0,0003)$, pero no así los aprendices pertenecientes a B1 y B2 $(\mathrm{t}=-1,05, \mathrm{p}=0.54)$.

Teniendo en cuenta que, según su actuación con respecto al instrumento manejado, solo hay dos grupos significativamente diferentes (uno de nivel B y otro de nivel C), examinaré por separado únicamente los resultados de dichos grupos. En la tabla 7 se presentan los índices de dificultad de los ítems para cada uno de estos dos grupos.

\begin{tabular}{lcccc|cccc}
\hline & \multicolumn{4}{c}{ Grupo B } & \multicolumn{4}{c}{ Grupo C } \\
\hline Ítems A & I1 & I8 & I15 & media & I1 & I8 & I15 & media \\
& 0,80 & 0,63 & 0,61 & 0,68 & 0,80 & 0,60 & 1 & 0,80 \\
\hline Ítems B1 & I2 & I6 & I13 & media & I2 & I6 & I13 & media \\
& 0,61 & 0,63 & 0,80 & 0,68 & 1 & 1 & 1 & 1 \\
\hline Ítems B2 & $\mathbf{I 4}$ & I7 & I11 & media & I4 & I7 & I11 & media \\
& 0,47 & 0,29 & 0,47 & 0,41 & 0,60 & 0,60 & 1 & 0,73 \\
\hline Ítems C1 & $\mathbf{I 5}$ & $\mathbf{I 9}$ & $\mathbf{I 1 2}$ & media & $\mathbf{I 5}$ & $\mathbf{I 9}$ & $\mathbf{I 1 2}$ & media \\
& 0,39 & 0,51 & 0,45 & 0,45 & 1 & 1 & 1 & 1 \\
\hline Ítems C2 & $\mathbf{I 3}$ & $\mathbf{I 1 0}$ & $\mathbf{I 1 4}$ & media & $\mathbf{I 3}$ & $\mathbf{I 1 0}$ & $\mathbf{I 1 4}$ & media \\
& 0,82 & 0,35 & 0,43 & 0,53 & 0,80 & 0,60 & 0,80 & 0,73 \\
\hline
\end{tabular}

Tabla 7. Índices de dificultad por grupos

Como en el caso de los resultados analizados en conjunto, es difícil advertir una progresión de la dificultad de los ítems en función de su nivel. Es cierto que en el caso de los aprendices de nivel $\mathrm{B}$ (el conjunto de participantes más numeroso) los ítems correspondientes a los niveles A1/A2 y B1 presentan proporciones medias de acierto más altas que el resto, pero el siguiente nivel más fácil en promedio es el C2 (el más alto). Asimismo, si bien es verdad que uno de los ítems más difíciles para este grupo pertenece al nivel C2 (el ítem 10), el que menos proporción de aciertos tiene (ítem 7) se sitúa en el nivel B2. Otro tanto puede decirse del grupo de nivel C: los participantes de este grupo hacen pleno en los niveles $\mathrm{B} 1$ y C1, mientras que las colocaciones que les resultan más difíciles se sitúan en el nivel B2 (como con el grupo B).

El análisis de los resultados relativos a aprendices presentado hasta aquí se basa en su clasificación en distintos niveles del MCER y la relación que dicha clasificación presenta con la proporción de aciertos obtenida para cada ítem. No obstante, para apreciar el efecto que la frecuencia de una colocación tiene en el grado de dificultad que dicha colocación presenta a un aprendiz, podría ser útil atender directamente a este factor dejando de lado la nivelación. Con tal propósito los resultados obtenidos con el cuestionario para aprendices se han analizado mediante un modelo mixto de regresión lineal (Gries 2015), usando como variable dependiente el acierto/error de cada ítem 
del cuestionario y como variables independientes el nivel de los aprendices y la frecuencia de cada colocación (efectos fijos) y teniendo en cuenta el efecto debido a la identidad de cada participante (efecto aleatorio). Los resultados se presentan en la tabla 8.

\begin{tabular}{lllll}
\hline & Estimación & Error estándar & valor-Z & $\mathrm{p}$ \\
\hline (Intersección) & $-0,32252$ & 0,17148 & 1,881 & 0,060004 \\
B2 & $-0,36214$ & 0,28463 & $-1,272$ & 0,203265 \\
C & 1,63040 & 0,51857 & 3,144 & $0,001667^{* *}$ \\
frecuencia & 0,28808 & 0,08295 & 3,473 & $0,000515^{* * *}$ \\
\hline
\end{tabular}

Tabla 8. Probabilidad de acierto según el rango de la colocación y el nivel del aprendiz (efectos fijos)

De manera similar a lo observado al analizar las diferencias entre los resultados de los aprendices de distintos niveles, únicamente las respuestas de los participantes de nivel C muestran una probabilidad significativamente más alta de acierto (las respuestas de los de B2 tienen una probabilidad de acierto más baja que las de los aprendices B1, pero el efecto no es significativo). En cuanto a la frecuencia de la colocación, se observa que un aumento en este parámetro conlleva un incremento significativo de la probabilidad de acierto. La proporción de la varianza explicada por el modelo en su totalidad es del 0,23, de la cual un 0,09 corresponde a los efectos fijos. Cabe pensar, pues, que la identidad de cada participante tiene un efecto más destacado en la probabilidad de acierto que el propio nivel de aprendizaje y la frecuencia colocacional y que hay más factores en juego que los considerados por este modelo.

\section{Discusión}

A continuación, intentaré justificar cómo se pueden interpretar los resultados presentados en el apartado precedente a la luz de las preguntas formuladas al inicio de este trabajo.

La primera de dichas preguntas hacía referencia a qué criterios emplearían los profesores de ELE/2 si tuvieran que nivelar una lista de colocaciones y, como se ha visto en el apartado anterior, la mayoría de los participantes optaron por la frecuencia de uso. La opción de escoger el vocabulario en función de los temas que se traten en cada nivel — que es, en principio, compatible con la anterior - aparece en tercer lugar, pero a bastante distancia.Estos resultados están en consonancia con la historia reciente de la enseñanza del vocabulario en el currículo de las LE/L2. Una síntesis de lo sucedido en este ámbito hasta la década de 1980 se presenta en Hindmarsh (1980: v-vi). El autor señala que tras una época de interés notable por los repertorios de léxico frecuente al menos hasta la mitad del siglo pasado ${ }^{5}$, a partir de la irrupción del paradigma generativista, la atención se dirigió de forma primordial hacia la gramática, dejando este tipo de repertorios como recur-

5 Hindmarsh se centra en el inglés, pero el interés por repertorios de léxico frecuente aplicados a la enseñanza de lenguas se verificó también en otras lenguas de nuestro entorno inmediato como testimonian el ya citado Keniston (1920) o, en el caso del francés, Le français élementaire (1954) y, posteriormente, el francés fundamental (vid. Gougenheim et al. 1964), donde el criterio de frecuencia se combinaba con los resultados de estudios de léxico disponible en hablantes nativos. 
sos obsoletos ${ }^{6}$. Siempre según Hindmarsh, la reacción a este paradigma por parte del enfoque sociolingüístico, que propugnaba centrarse en los propósitos comunicativos de los hablantes, las funciones puestas en práctica para servir tales propósitos y la relación con el contexto, no significó necesariamente una vuelta al estudio de la frecuencia léxica, en la medida en que la atención a los citados factores no implica directamente una concreción en cuanto a qué contenidos lingüísticos corresponden a cada nivel del proceso de aprendizaje. En palabras del citado autor:

Purpose, context, tone, mode and medium can be orchestrated to produce a language specification: but that specification can - and I maintain must - be graded in some measure to make it right for where the learner is now. (Hindmarsh 1980: vi)

Entre el panorama descrito por Hindmarsh y la situación presente parece haber resurgido la idea de que los contenidos léxicos merecen atención en sí mismos (incluso que pueden vertebrar la totalidad de los que se han de tratar en la enseñanza de una LE/L2, cf. Lewis 1993) y que la frecuencia de uso puede ser un criterio valioso a la hora de seleccionarlos y secuenciarlos (Martinez 2013; Nation 2001: 13 y ss., 329; Sinclair y Renouf 1985). En lo que respecta al español y, si consideramos el PCIC como un documento representativo de la práctica de los docentes de esta lengua, el enfoque nocio-funcional sigue presente y los contenidos léxicos (en un sentido amplio, que incluye la fraseología) y de otro tipo están, por una parte, supeditados a la organización en funciones y nociones que se prevé para cada nivel, pero, por otra, se reconoce de forma explícita (Introducción a las Nociones específicas) que los exponentes seleccionados lo han sido en función de su "frecuencia y rentabilidad comunicativa".

En lo que concierne a las preguntas ii) y iii), relativas a la intuición sobre frecuencia colocacional de los profesores participantes y a la coincidencia entre la nivelación que ellos proponen y una basada puramente en frecuencia de uso, los resultados presentados en el apartado anterior parecen llevarnos a respuestas contradictorias. Recuérdese que, cuando se les pide proponer una nivelación de una lista de colocaciones, dicha nivelación presenta en aproximadamente dos tercios de los participantes un coeficiente de correlación de al menos 0,60 con la nivelación del DiCE (basada exclusivamente en la frecuencia, como se ha dicho ya). Recuérdese también que la tarea pedía simplemente nivelar un listado de acuerdo con los niveles del MCER, pero no mencionaba ningún criterio en concreto y las cuestiones donde se citaba la frecuencia de uso aparecían más tarde en el cuestionario. Ahora bien, cuando se solicita directamente que los mismos participantes ordenen un listado de colocaciones de la más a la menos frecuente, menos de una quinta parte obtiene una correlación de 0,60 o más entre la ordenación propuesta y la lista ordenada por frecuencia. Además, el promedio de los coeficientes obtenidos es considerablemente más bajo que en la otra tarea comentada (vid. supra). Es decir, pocos de los profesores participantes en el estudio tienen intuiciones fiables con respecto a la frecuencia de las colocaciones y, a pesar de ello, la mayoría nivela de forma intuitiva un listado de colocaciones de acuerdo con su frecuencia, lo cual resulta paradójico.

6 En el mundo anglosajón solo relativamente, pues las listas de frecuencia no han dejado de usarse desde la década de 1960 para controlar el léxico que se incluye en las lecturas graduadas. En español, sin embargo, no deja de ser sorprendente que para nivelar este tipo de textos parece haber primado el criterio gramatical hasta fecha reciente (cf. Báez Montero y Suárez Rodríguez 2012: 122; o San Mateo Valdehíta 2005: 41). 
Parte de la paradoja probablemente se deba a las diferencias entre las dos tareas. En primer lugar, en la tarea de nivelación los niveles del MCER se han agrupado en cinco categorías, mientras que en la lista para ordenar por frecuencia los participantes tienen nueve colocaciones, de manera que en el primer caso, en la práctica, solo hay cinco rangos posibles — aunque la lista contenga diez ítems-, mientras que en el otro hay nueve, lo que incrementa la dificultad de la prueba. En segundo lugar, como se ha indicado arriba, en la tarea de ordenar por frecuencia se ha procurado, siguiendo a McGee (2006) que cada colocación sea aproximadamente el doble de frecuente que la siguiente en la lista. En la tarea de nivelación, la frecuencia no se controló, lo cual daba lugar a que hubiese diferencias más extremas. Así, la colocación menos frecuente del nivel A1/A2 se presenta en el corpus alrededor de diez veces más (tener [la] culpa, con 6,41 ocurrencias por millón de palabras) que la más frecuente del B1 (mantener [una/la] amistad, 0,60 ocurrencias). En la frontera entre los dos niveles siguientes las distancias se acortan algo y la colocación menos común de B1 (perder el respeto, 0,56) es aproximadamente tres veces más frecuente que la que más ocurrencias presenta en B2 (causar admiración, 0,19). A partir de aquí las diferencias entre niveles en cuanto a frecuencia decrecen y son similares a las de la otra tarea discutida.

Los resultados en cuanto a la intuición de los participantes también contrastan con los obtenidos para el inglés por McGee (2006:137). El autor, en una prueba de carácter similar a la comentada, obtiene medias de coeficientes de correlación (Spearman) que van del 0,53 al 0,85 y en tres de las cuatro listas que presenta a sus participantes, las medias superan el 0,70 - recuérdese que en el presente estudio, las medias oscilan entre el 0,45 y el 0,29, dependiendo de que se considere que quienes han obtenido coeficientes negativos han ordenado la lista a la inversa de como se les solicitaba o no-. No obstante, los resultados obtenidos aquí no son directamente comparables con los de McGee (2006) por dos motivos. En primer lugar, la tarea de ordenación por frecuencia discutida aquí iba dirigida a profesores de español y no se ha controlado que fuesen o no hablantes nativos. El experimento de McGee, por el contrario, se centraba exclusivamente en hablantes nativos del inglés. En segundo lugar, en las listas presentadas por McGee, formadas por combinaciones de nombre más adjetivo, uno de los elementos (el adjetivo) se mantenía constante (por ejemplo, una de ellas era una serie de colocaciones con el adjetivo personal, otra consistía en colocaciones con el adjetivo bad, etc.). Por el contrario, la lista propuesta a los participantes de este estudio incluía nueve colocaciones verbo-objeto constituidas por lemas diferentes. Se podría pensar, pues, que en el caso de McGee los participantes están nivelando nombres (los elementos variables en cada una de las listas) y no colocaciones, aunque el autor se cuida de asegurarse que las diferencias de frecuencia en cada lista no se deban exclusivamente al nombre (las correlaciones entre la frecuencia de los nombres y la de las respectivas colocaciones no resultan significativas; McGee 2006: 130-133). En cualquier caso, llaman la atención la similitud entre las medias de coeficientes obtenidas por McGee y las de estudios centrados en intuiciones sobre palabras aisladas ${ }^{7}$, de una parte, y el contraste con los resultados obtenidos aquí, de otra.

Por último, por lo que respecta a los aprendices, los resultados parecen sugerir que una nivelación basada exclusivamente en la frecuencia no nos dice mucho acerca de la relación entre la pertenencia de una colocación a un determinado nivel y el grado de dificultad que presenta.

7 Arnaud (1990) obtiene correlaciones medias de entre el 0,61 y el 0,76, dependiendo de la lengua materna de los hablantes y de la lengua testada; Frey (1981), entre 0,91 y 0,95, dependiendo de la lista de frecuencia utilizada; Ringeling (1984), entre 0,68 y el 0,79 dependiendo del grupo de hablantes. 
La correlación entre la facilidad de los ítems y el nivel va en la dirección esperada (a mayor nivel, menor proporción de aciertos), pero es pequeña y no resulta significativa. Si nos fijamos en el grupo de participantes mayoritario (los pertenecientes a B1 y B2), parece haber una brecha entre los ítems de los dos niveles más bajos (A1/A2 y B1) y el resto. Los primeros son los que presentan una mayor proporción de aciertos — son los más fáciles- y son también los más frecuentes de todo el conjunto. En los segundos se da una tendencia inversa con respecto a lo que cabría esperar: $\mathrm{C} 2$ presenta en promedio más aciertos que $\mathrm{C} 1$ y este nivel más que B2. Ahora bien, entre los ítems de C2 hay uno que claramente se destaca por haber recibido un número de aciertos mucho mayor (una proporción del 0,82 ) que los otros representantes de su nivel y de los niveles de B2 a C2 en general (el correspondiente a la colocación expresar aprecio). Asimismo, en B2 se incluye uno con una proporción considerablemente menor que el resto (levantar el ánimo). Además, cuando se deja de lado la nivelación y se considera la frecuencia de cada colocación de forma individualizada y su efecto en la probabilidad de acierto en cada una de las respuestas ( $v i d$. tabla 8 supra), se obtiene que aquella es un elemento facilitador (por pequeño que sea su efecto), lo cual podría considerarse una evidencia más de que la presencia de determinados ítems distorsiona la relación que cabría esperar entre nivel y dificultad. Esto puede cuestionar dos aspectos del instrumento: por una parte, su extensión y, por otra, la formulación de los propios ítems.

En cuanto a la extensión, cabe apuntar que la presencia de valores atípicos entre los resultados de cada nivel evidentemente tendría efectos más discretos si el número de ítems por nivel fuese mayor. Esto lleva también a cuestionarse la representatividad de las colocaciones elegidas para cada uno de los niveles. El repertorio de colocaciones que se recoge en el DiCE es muy abundante (alrededor de 15000), aun limitándose por el momento a un solo campo semántico. La limitada representatividad de tres ítems por nivel se hace más acusada en las colocaciones de frecuencia baja (y por tanto, con niveles altos), que son el conjunto más numeroso del diccionario. Ahora bien, la viabilidad de un instrumento mucho más extenso es dudosa, teniendo en cuenta el carácter voluntario de la participación en el estudio, así como el tiempo disponible de los participantes.

En cuanto a la formulación de los ítems, tal como se justifica en el apartado 2, la necesidad de controlar la producción de los participantes condujo al formato de respuesta múltiple.Ahora bien, este formato supone que se han de incorporar elementos adicionales (distractores) que afectan la respuesta de los participantes. En el caso concreto de expresar aprecio es muy difícil determinar en qué medida un participante determinado conoce la colocación meta o la elige descartando los distractores que se le ofrecen como alternativa.

En definitiva, los resultados relativos a los aprendices obtenidos aquí han de tomarse con precaución y contrastándolos con datos obtenidos por medio de otro tipo de métodos, como los empleados en lingüística de corpus, para obtener una mejor comprensión del fenómeno estudiado (cf. Alonso Ramos 2016: 15; Ellis y Barkhuizen 2005: 49). En este sentido, cabe apuntar que cuando se examina la producción libre de colocaciones por parte de aprendices de una L2/LE, normalmente se suele observar que estos hacen un mayor uso de combinaciones frecuentes que los hablantes nativos (Durrant y Schmitt 2009; Vincze et al. 2016) o que aprendices más avanzados (Granger y Bestgen 2014), hasta el punto que algunas colocaciones de frecuencia muy alta parecen actuar como una especie de comodín. Inferir a partir de este tipo de datos de uso que las colocaciones más frecuentes se adquieren antes o con más facilidad que las menos frecuentes puede 
suponer un salto injustificado en el razonamiento, en la medida que el uso en producción libre solo nos puede ofrecer un panorama muy parcial del conocimiento de un hablante con respecto al objeto estudiado. Si bien la metodología de obtención de datos a través de producción controlada empleada aquí puede ser un complemento valioso, en la medida en que brinda un acceso en teoría más inmediato a la competencia de los hablantes en un determinado fenómeno, introduce también elementos distorsionadores, como se acaba de señalar.

Finalmente, más allá de la influencia que hayan podido tener los instrumentos de recogida de datos en los resultados, parece evidente que hay otros factores independientes de la frecuencia de uso que inciden en la dificultad que una colocación pueda tener para un aprendiz y, en consecuencia, en la necesidad de incluirla de manera explícita en una u otra fase del proceso de aprendizaje. Probablemente el más evidente a este respecto es la lengua materna del aprendiz, elemento que alguno de los profesores participantes en el cuestionario ha incluido como criterio nivelador, junto a la dificultad. Además, los propios intereses de los aprendices, así como sus distintas trayectorias de aprendizaje probablemente hayan influido en el léxico al que han estado expuestos.

\section{Conclusiones}

El trabajo presentado es una aproximación inicial al estudio de la validez de la frecuencia de uso como criterio nivelador del vocabulario en la enseñanza de ELE/2 y parte de una muestra pequeña y no del todo equilibrada, por lo que se hacen necesarias ulteriores investigaciones con muestras más amplias. Aún siendo conscientes de ello, de él se deprende que la frecuencia de uso, tanto desde el punto de vista teórico, como en opinión de los profesionales de la enseñanza de español, es un criterio fundamental para nivelar las colocaciones que se han de presentar a los aprendices. No se ha podido demostrar, sin embargo, que un sistema de nivelación basado exclusivamente en la frecuencia se corresponda con el grado de dificultad que presentan las colocaciones a los aprendices — al menos en una tarea de reconocimiento-. Probablemente factores como la lengua materna de cada aprendiz, sus diversas trayectorias de aprendizaje, etc., inciden en dicha dificultad y los profesionales de la enseñanza son conscientes de ello. Ahora bien, la atención a dichos factores individuales en obras que no se dirijan específicamente a aprendices con una determinada L1, como, por ejemplo, un diccionario de colocaciones, no parece tener cabida. Por ello, la frecuencia de uso parece un buen criterio de partida. La atención a criterios tales como la disponibilidad léxica en hablantes nativos (Benigno et al. 2015), la relación con las áreas temáticas tratadas en cada nivel (Spina 2016), etc., no se han tratado aquí y merecerían investigaciones aparte.

Además, el estudio de los factores que condicionan la dificultad de una colocación por parte de un aprendiz requiere una mayor profundización. Por una parte, en el presente trabajo solo se ha podido prestar atención a la dificultad de reconocer una determinada colocación del español en un contexto dado (limitación impuesta por la necesidad de controlar la respuesta de los participantes), pero el conocimiento colocacional de un aprendiz se compone de más dimensiones (producción, comprensión) que deberían ser abordadas. Por otra parte, como se comenta en la discusión, tanto el modelo de regresión al que se sometieron los resultados del cuestionario a aprendices, como ciertos trabajos que analizan textos de producción libre, apuntan a un papel 
facilitador de la frecuencia de uso, lo que en cierto modo contrasta con los resultados obtenidos aquí con respecto a la nivelación.

Volviendo al papel de los profesionales de la enseñanza, se ha visto que si bien hay un amplio consenso en cuanto a la importancia de la frecuencia de uso para determinar qué colocaciones deben tratarse en un determinado nivel de aprendizaje, probablemente la intuición de dichos profesionales no sea suficiente cuando se trata de nivelar. Los propios participantes parecen abiertos a usar materiales de consulta donde el vocabulario se presente ya nivelado (de hecho, esta es la segunda opción más frecuente cuando se les pregunta por criterios de nivelación; vid. apartado 2). En este sentido, cabe recordar que existe una gran variabilidad entre los hablantes en cuanto a su intuición sobre frecuencia colocacional y los que presentan correlaciones altas con respecto al corpus manejado son minoría. Podría cuestionarse aquí qué recurso es más representativo de la experiencia lingüística de un hablante: si un determinado corpus, que podría estar mal concebido o sesgado hacia un determinado tipo de discurso, o su propia intuición, en tanto que está expuesto diariamente a muestras de lengua natural. Con todo, habida cuenta de la discrepancia entre los propios hablantes, un corpus parece un recurso mucho más objetivo en este sentido. En cualquier caso, parece claro que si la frecuencia de uso es un elemento de juicio razonable para determinar la nivelación del vocabulario, se hacen necesarios repertorios (diccionarios, listados, planes docentes, etc.) que contengan información al respecto procedente de corpus. Algunos de los profesionales consultados en el estudio señalan que este debería ser el camino a seguir: así, en la tarea de ordenar colocaciones de mayor a menor frecuencia, uno de ellos apunta que sería necesaria la consulta de un corpus para llevarla a cabo de manera fiable.

Por último, cabe notar que la correspondencia entre las respuestas de los participantes y la frecuencia de uso variaba dependiendo de la tarea. Asimismo, la pobre intuición sobre frecuencia observada en el estudio del que se da cuenta aquí contrasta con investigaciones anteriores referidas a otras lenguas. Por todo ello, las intuiciones sobre frecuencia colocacional de los hablantes de español merecerían también atención en futuras investigaciones.

\section{Referencias bibliográficas}

Alonso Ramos, M. (2004). Diccionario de colocaciones del español. <http://www.dicesp.com/>

. (2012). Explorando la frecuencia léxica para el Diccionario de colocaciones del español. In T. Jiménez Juliá, B. López Meirama, V. Vázquez Rozas, \& A. Veiga (Eds.), Cum corde et in nova grammatica. Estudios ofrecidos a Guillermo Rojo (pp. 19-40). Santiago de Compostela: Universidade de Santiago de Compostela.

- (2016). Spanish learner corpus research: Achievements and challenges. In M. Alonso Ramos (Ed.), Spanish Learner Corpus Research. Current trends and future perspectives (pp. 3-32). Amsterdam/Philadelphia: John Benjamins.

Arnaud, P. J. L. (1990). Subjective word frequency estimates in LI and L2. Paper presented at the 9th World Congress of Linguistics, Thessaloniki. ERIC Document ED329120, 1-15.

Báez Montero, I., \& Suárez Rodríguez, B. (2012). Lectura analógica / lectura digital : 'el papel' de las lecturas graduadas en aprendices de E / LE. In C. Hernández González, A. Carrasco Santana \& E. Álvarez 
Ramos (Eds.), La Red y sus aplicaciones en la enseñanza-aprendizaje del español como lengua extranjera (pp. 117-128). Valladolid: ASELE.

Benigno, V., Kraiff, O., Grossmann, F., \& Velez., A. (2016). La notion de collocation fondamentale: une étude de corpus. Cahiers de Lexicologie, 108(1), 125-146.

Capel, A. (2010). A1 - B2 vocabulary: insights and issues arising from the English Profile Wordlists project. English Profile Journal, 1(1), e3.

- (2012). Completing the English Vocabulary Profile: C1 and C2 vocabulary. English Profile Journal, 3 (June 2012), e1.

Consejo de Europa. (2001). Marco Común Europeo de Referencia para el aprendizaje de lenguas: aprendizaje, enseñanza, evaluación. Madrid: Ministerio de Educación, Cultura y Deporte.

Davies, M. (2006). A frequency dictionary of Spanish. New York/London: Routledge.

Dörney. Z. (2002). Questionnaires in Second Language Research. London: Erlbaum.

Durrant, P., \& Schmitt, N. (2009). To what extent do native and non-native writers make use of collocations? IRAL - International Review of Applied Linguistics in Language Teaching, 47(2), 157-177.

Ellis, R., \& Barkhuizen, G. (2005). Analysing Learner Language. Oxford: Oxford University Press.

Ferrando Aramo, V. (2012). Aspectos teóricos y metodológicos para la compilación de un diccionario combinatorio destinado a estudiantes de E/LE. Tesis doctoral. Universitat Rovira i Virgili, Tarragona.

Frey, E. (1981). Subjective word frequency estimates and their stylistic relevance in literature. Poetics, 10(4-5), 395-407.

García Salido, M., \& Alonso Ramos, M. (en prensa). Asignación de niveles de aprendizaje a las colocaciones del Diccionario de Colocaciones del Español. Revista Signos.

Gilquin, G. (2007). To Err Is Not All: What Corpus and Elicitation Can Reveal About the Use of Collocations By Learners. Zeitschrift für Anglistik und Amerikanistik, 55(3), 273-291.

Gougenheim, G., Michéa, R. Rivenc, P., \& Sauvageot, A. (1964). Lélaboration du français fundamental. Paris: Didier.

Granger, S., \& Bestgen, Y. (2014). The use of collocations by intermediate vs. advanced non-native writers: A bigram-based study. IRAL - International Review of Applied Linguistics in Language Teaching, 52(3), 229-252.

Gries, S. T. (2015). The most under-used statistical method in corpus linguistics: Multi-level (and mixedeffects) models. Corpora, 10(1), 95-125.

Herberich, E., Sikorski, J., \& Hothorn, T. (2010). A robust procedure for comparing multiple means under heteroscedasticity in unbalanced designs. PLoS ONE, 5(3), 1-8.

Instituto Cervantes. (1997-2016). Plan Curricular del Instituto Cervantes. $<$ http://cvc.cervantes.es/Ensenanza/biblioteca_ele/plan_curricular/default.htm>

Izquierdo, M. C. (2004). La selección del léxico en la enseñanza del español como lengua extranjera. Su aplicación en el nivel elemental en estudiantes francófonos. Valencia: Serveix de de Publicacions, Universitat de Valencia.

Keniston, H. (1920). Common words in Spanish. Hispania, 3, 85-96.

Kilgarriff, A., \& Renau, I. (2013). esTenTen, a Vast Web Corpus of Peninsular and American Spanish. Procedia - Social and Behavioral Sciences, 95, 12-19.

Laufer, B., \& Goldstein, Z. (2004). Testing vocabulary knowledge: Size, strength, and computer adapativeness. Language Learning, 54(3), 399-436.

Lewis, M. (1993). The Lexical Approach. London: Language Teaching Publications.

Martinez, R. (2013). A framework for the inclusion of multi-word expressions in ELT.ELT Journal, 67(April), 184-198.

McGee, I. D. (2006). Lexical Intuitions and Collocation Patterns in Corpora. Tesis doctoral. Cardiff University. 
Mel'cuk, I. (2012). Phraseology in the language, in the dictionary, and in the computer. Yearbook of Phraseology, 3, 31-56.

Nation, P. (2001). Learning vocabulary in another language. Cambridge: Cambridge University Press.

Nesselhauf, N. (2004). Collocations in a Learner Corpus. Amsterdam/Philadelphia: John Benjamins.

Ringeling, T. (1984). Subjective estimations as a useful alternative to word frequency counts. Interlanguage Studies Bulletin, 20(8), 59-69.

San Mateo Valdehíta, A. (2005). Una lectura graduada narrativa para estudiantes de ELE de nivel avanzado. Tesis de máster. <http://www.mecd.gob.es/redele/Biblioteca-Virtual/2005/memoriaMaster/1-Semestre/ SAN-MATEO-UCM.html>

Schmitt, N., \& Dunham, B. (1999). Exploring native and non-native intuitions of word frequency. Second Language Research, 15, 389-411.

Spina, S. (2016). Learner Corpus Research and phraseology in Italian as a second language: the case of the DICI-A, a learner dictionary of Italian collocations. In B. Sanromán Vilas (Ed.), Collocations Cross-Linguistically. Corpora, Dictionaries and Language Teaching (pp. 219-244). Helsinki: Mémoires de la Société Néophilologique de Helsinki.

Vincze, O., \& Alonso Ramos, M. (2013). Incorporating Frequency Information in a Collocation Dictionary: Establishing a Methodology. Procedia - Social and Behavioral Sciences, 95, 241-248.

Vincze, O., García Salido, M., Orol, A., \& Alonso Ramos, M. (2016). A corpus study of Spanish as a Foreign Language learners' collocation production. In M. Alonso Ramos (Ed.), Spanish Learner Corpus Research. Current trends and future perspectives (pp. 209-332). Amsterdam/Philadelphia: John Benjamins. 
